\title{
Identifying global expression patterns and key regulators in epithelial to mesenchymal transition through multi-study integration
}

\author{
Princy Parsana', Sarah R. Amend², James Hernandez ${ }^{2}$, Kenneth J. Pienta ${ }^{2}$ and Alexis Battle ${ }^{1 *}$
}

\begin{abstract}
Background: Epithelial to mesenchymal transition (EMT) is the process by which stationary epithelial cells transdifferentiate to mesenchymal cells with increased motility. EMT is integral in early stages of development and wound healing. Studies have shown that EMT could be a critical early event in tumor metastasis that is involved in acquisition of migratory and invasive properties in multiple carcinomas.

Methods: In this study, we used 15 published gene expression microarray datasets from Gene Expression Omnibus (GEO) that represent 12 cell lines from 6 cancer types across 95 observations (45 unique samples and 50 replicates) with different modes of induction of EMT or the reverse transition, mesenchymal to epithelial transition (MET). We integrated multiple gene expression datasets while considering study differences, batch effects, and noise in gene expression measurements. A universal differential EMT gene list was obtained by normalizing and correcting the data using four approaches, computing differential expression from each, and identifying a consensus ranking. We confirmed our discovery of novel EMT genes at mRNA and protein levels in an in vitro EMT model of prostate cancer - PC3 epi, EMT and Taxol resistant cell lines. We validate our discovery of C1orf116 as a novel EMT regulator by siRNA knockdown of C1orf116 in PC3 epithelial cells.
\end{abstract}

Results: Among differentially expressed genes, we found known epithelial and mesenchymal marker genes such as CDH1 and ZEB1. Additionally, we discovered genes known in a subset of carcinomas that were unknown in prostate cancer. This included epithelial specific LSR and S100A14 and mesenchymal specific DPYSL3. Furthermore, we also discovered novel EMT genes including a poorly-characterized gene C1orf116. We show that decreased expression of C1orf116 is associated with poor prognosis in lung and prostate cancer patients. We demonstrate that knockdown of Clorf116 expression induced expression of mesenchymal genes in epithelial prostate cancer cell line PC3-epi cells, suggesting it as a candidate driver of the epithelial phenotype.

Conclusions: This comprehensive approach of statistical analysis and functional validation identified global expression patterns in EMT and candidate regulatory genes, thereby both extending current knowledge and identifying novel drivers of EMT.

Keywords: EMT, Metastasis, Prostate cancer, C1orf116, Multi-study integration

\footnotetext{
* Correspondence: ajbattle@cs.jhu.edu

${ }^{1}$ Department of Computer Science, Johns Hopkins University, Baltimore, MD

21218, USA

Full list of author information is available at the end of the article
} 


\section{Background}

Cancer is the second leading cause of death in United States. Metastasis is the leading cause of cancer-related morbidity and mortality [1], but identifying tumors with metastatic potential remains a challenge [2]. Tumor metastasis is a multi-step process in which primary tumor cells disseminate from their site of origin to seed secondary tumors at a distant site [3]. It is believed that in a critical early event in cancer progression, metastatic cancer cells undergo an epithelial to mesenchymal transition (EMT). During EMT, stationary epithelial cells lose cell polarity and transdifferentiate to spindle-shaped motile mesenchymal cells. EMT is a crucial physiologic process involved in early development during embryogenesis and organogenesis. It also plays an important role in tissue regeneration and wound healing. However, in cancer EMT may contribute to tumor progression and malignant transformation. Several epithelial cancer cells have been described to undergo EMT transform to a more malignant phenotype [4] that can further promote formation of secondary tumors [5].

The role of EMT has been frequently debated in clinical cancer metastasis [6]. However, several in vitro studies have shown that epithelial cancer cells can undergo EMT in response to a combination of signals from the tumor microenvironment [2]. During EMT, cells go through multiple morphological and biochemical changes resulting in loss of epithelial properties coupled with gain of mesenchymal characteristics [7-21]. Microarrays have been widely used to study gene expression patterns of cell populations under different experimental settings, including EMT-inducing conditions (Fig. 1). While there have been many studies investigating the effect of a gene or pathway in EMT, none have explored the universal changes across multiple cancer tissue types or EMT induction methods.

Several gene expression datasets examining EMT in a variety of different cell lines under different conditions are available on open access databases such as Gene Expression Omnibus (GEO) [22]. It has been demonstrated that re-use and aggregation of public gene expression data facilitates discovery of signals too weak to be detected in an individual experiment [23-26]. Gröger et al. performed meta-analysis of 18 EMT gene expression studies and identified 130 core-EMT genes, which were differentially expressed in at least 10 of the 18 studies [27]. Genes such as TGFB, GNG11, TIMP1, ETS1, S100A14, DPYSL3 and C1orf116 that we discovered as differential EMT, were not found in their core EMT gene list. Furthermore, we experimentally validated some of these genes (S100A14, DPYSL3 and C1orf116) in PC3 epithelial, PC3-EMT and PC3-taxol resistant cell lines confirming their association in EMT. Also, each dataset in [27] was confined by small sample size per class $(\mathrm{n}<=6)$. The drawback with underpowered studies are: a) low probability of identifying true effects b) overestimation of effect size $[28,29]$. Therefore, genes that showed consistent moderate effects across datasets could be missed. In contrast, systematic integration of multiple studies promotes reliable detection of consistent gene expression changes that may otherwise be false negatives in results obtained from individual experiment [30]. At the same time, it helps avoid false discoveries that could result from intra-study variability resulting from single experiment.

Batch effects and noise introduce spurious signal and correlations in microarray gene expression data [31, 32]. Therefore, data normalization is crucial in order to correct the data for unwanted biological or non-biological effects. However, Groger et al. do not account for batch effects, cross-platform differences, or cross-tissue effects in their meta-analyses study that could potentially lead to false positive findings.

In this study, to identify universal EMT genes common across multiple cancer types, we integrated 15 independent gene expression studies representing 12 cell lines (49

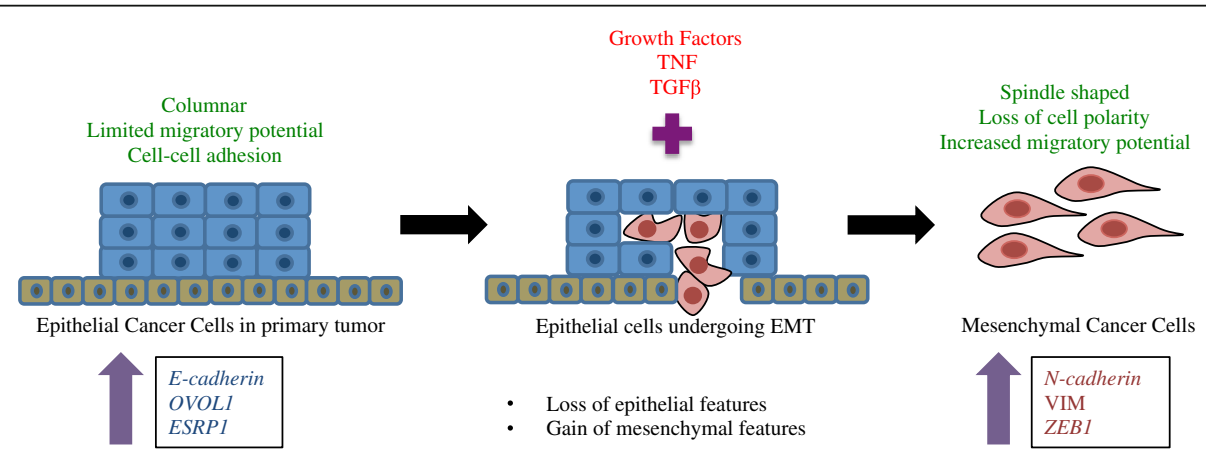

Fig. 1 Epithelial to Mesenchymal Transition. During EMT, non-motile epithelial cells trans differentiate to mesenchymal cells with increased migratory potential. During this, cells show decreased expression epithelial specific genes that include E-cadherin, OVOL1 and ESRP1. At the same time, expression of mesenchymal genes such as N-cadherin, VIM and ZEB1 increases 
epithelial and 46 mesenchymal phenotypes) from 6 cancer tissue types and multiple EMT induction modalities (Table 1, Additional file 1: Table S1). After correcting data to account for cross-study differences, crossplatform differences, and other sources of noise, we performed differential expression analysis and identified global changes in gene expression patterns between epithelial and mesenchymal states (Fig. 2). Importantly, our candidate gene list was enriched for EMT-related genes and we identified known markers of EMT. In addition, we also identified EMT genes that had only been described in a sub-set of malignant disease states, but were previously unknown in prostate cancer (e.g. LSR, S11A14, DPYSL3), implying a common EMT program across multiple cancer types. We further identified genes that had not been previously characterized in EMT in any disease state including C1orf116, which we then experimentally validated using siRNA knockdown in PC3 epithelial cells. This approach of multi-study integration enabled identification of differential EMT genes universal across different types of cancer. Functional validations of these genes indicate manifestation of molecular mechanisms contributing to EMT shared across disease types. This study also identifies an uncharacterized candidate novel EMT regulator gene C1orf116. These findings thereby extend our knowledge and understanding of EMT biology.

\section{Methods}

\section{Data overview}

We used 15 published EMT microarray gene expression datasets from GEO (Gene Expression Omnibus) (Table 1, Additional file 1: Table S1). This comprises of 95 observations (45 unique samples and 50 replicates), 49 epithelial and 46 mesenchymal cell lines exposed to different treatment modalities. The cell lines come from 6 different tissue types including breast, prostate, colon, esophageal, liver and retinal pigment and 4 different microarray platforms (8 chips), Affymetrix, Agilent, Stanford Microarray Database (SMD) and Illumina. All the datasets were downloaded in the format they were submitted to GEO. We mapped platform specific probe IDs to Ensembl IDs and gene symbols. When multiple probes mapped to same gene, we used median values to represent expression of that gene. We used 7276 genes common across all datasets.

\section{Data normalization}

This work combined data from multiple studies spanning diverse cell lines and different platforms. Batch effects and noise are inherent in gene expression data. To account for confounders in data as a result of crossstudy and cross-platform effects, we used multiple correction methods, such as quantile normalization (QN), Surrogate Variable Analysis (SVA), Quantile normalization followed by SVA and Column Standardized Median Centered (MCtr). We merged all 15 datasets into one matrix prior to quantile normalization and SVA. For CMSC, we individually processed each study and combined them after normalization.

\section{Quantile normalization}

Quantile normalization makes the gene expression distribution of each sample in the dataset the same. Given a dataset D, with 'g' genes and 'n' samples:

Table 1 Dataset information

\begin{tabular}{|c|c|c|c|c|c|}
\hline GEO ID & Platform ID & Disease Type & Cell line & Samples* & $\overline{\operatorname{Ref}}$ \\
\hline GSE12811 & GPL7319 & Breast & MCF10A & 3 & {$[7]$} \\
\hline GSE13915 & GPL7785 & Breast & BT549, EFM19 & 4 & {$[8]$} \\
\hline GSE18070 & GPL570 & Breast & MCF10CA1h & 9 & [9] \\
\hline GSE28569 & GPL6480 & Breast & MCF10A & 8 & [10] \\
\hline GSE39356 & GPL6480 & Breast & MCF-7 & 4 & [11] \\
\hline GSE8240 & GPL3921 & Breast & MCF10A & 11 & [12] \\
\hline GSE12203 & GPL2700 & Colon & Caco-2 & 4 & [13] \\
\hline GSE14773 & GPL570 & Colon & HT29, SW480 & 8 & [14] \\
\hline GSE27424 & GPL570 & Esophageal & EPc2-hTERT & 12 & [15] \\
\hline GSE26391 & GPL6244 & Liver & HCC-1.1, HCC-1.2 & 8 & [16] \\
\hline GSE14405 & GPL570 & Prostate & PC3, TEM4, TEM2 & 6 & [17] \\
\hline GSE22010 & GPL6244 & Prostate & PrEC-hTERT & 2 & [18] \\
\hline GSE22764 & GPL6884 & Prostate & PC3 & 6 & [19] \\
\hline GSE43489 & GPL570 & Prostate & PC3 & 4 & [20] \\
\hline GSE12548 & GPL570 & Retinal pigment & ARPE19 & 6 & [21] \\
\hline
\end{tabular}

*Indicates the number of samples included in our study 


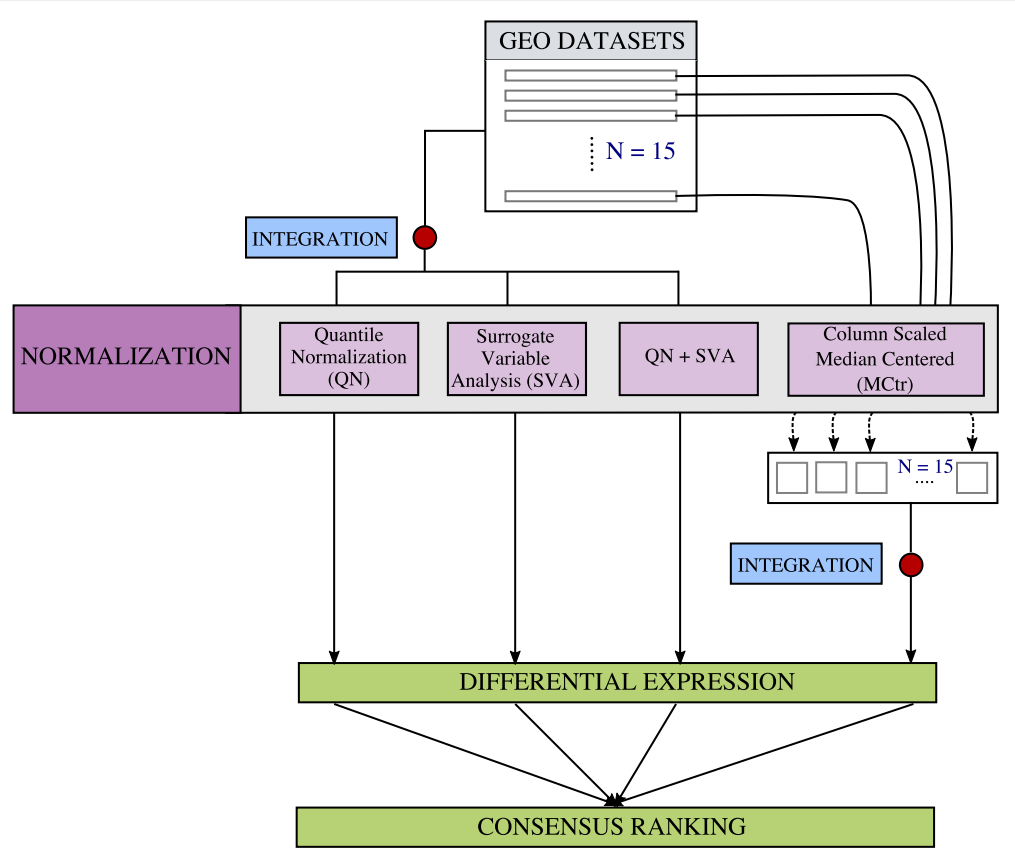

Fig. 2 Workflow for multi-study data integration, normalization and identification of candidate universal EMT genes

- Sorts each column in D

- Computes mean for each row and assigns it to each element in the row giving D'

- Finally, it rearranges columns in D' such that it has the same ordering as original $\mathrm{D}$, thus giving normalized data, D normalized

At the end of this, each column in D has the same distribution [33].

\section{Surrogate variable analysis}

Surrogate variable analysis allows us to preserve the phenotype signal of interest (epithelial and mesenchymal). It estimates known and hidden confounding factors using Singular Value Decomposition on residual variation matrix. We regress out estimated surrogate variables from gene expression data to get SVA normalized gene expression [34].

We also quantile normalize combined data followed by SVA to correct for hidden confounders.

\section{Column standardized median centered}

Samples from each study are standardized and median centered by gene as described in [35] and combined them.

\section{Differential expression analyses and concordance between} normalization methods

With each of the normalized dataset, we used a twosample $\mathrm{t}$-test to identify differentially expressed genes between epithelial and mesenchymal states. Assuming equal variance, we compared the mean expression of a gene between the two populations. For each gene, we tested:

Null Hypothesis : $\mu_{e p i}=\mu_{m e s}$

Alternative : $\mu_{\text {epi }} \neq \mu_{\text {mes }}$

We ranked genes by raw $p$-values. We applied Bonferroni correction for multiple hypothesis testing.

To test concordance between normalization methods, we used spearman rank correlation to test association between gene ranks $(n=7276)$ obtained by different correction methods.

Assuming equal probability of error for each normalization method, we computed average rank for each gene across the four methods that represented the consensus position of each gene according to the differential expression test statistic (Fig. 2).

\section{Cluster evaluation of normalized data}

To evaluate if normalization improved overall grouping of epithelial and mesenchymal phenotypes together, we clustered each of the normalized data using hierarchical clustering (with all 7276 genes). Next, to evaluate grouping we used Baker Hubert Index for cluster evaluation. Baker Hubert's Index (BH) [36] is an adaptation of Goodman and Kruskal gamma statistic in the context of clustering. 


$$
B H=\frac{S^{+}-S^{-}}{S^{+}+S^{-}}
$$

Here, $S^{+}$is the number of concordant quadraples and $S^{-}$is the number of disconcordant quadraples. To compute $\mathrm{BH}$, it tests all possible quadraples in the input.

Suppose we were testing quadruple samples $a, b, c, d$. And $d(a, b)$ is the distance between samples $a$ and $b$. A quadruple is concordant if it fulfills one of the following two conditions:

- $d(a, b)>d(c, d)$; And c and d are in same cluster and $\mathrm{a}$ and $\mathrm{b}$ are in different clusters

- $d(a, b)<d(c, d)$; And a and b are in same cluster and $c$ and $d$ are in different clusters

A quadruple is disconcordant if:

- $d(a, b)>d(c, d)$; And a and b are in same cluster and $c$ and $\mathrm{d}$ are in different clusters

- $d(a, b)<d(c, d)$; And $c$ and $d$ are in same cluster and $a$ and $b$ are in different clusters

Since we were interested in improvement in grouping of epithelial and mesenchymal samples, we used phenotype vector as cluster assignment for evaluation.

\section{Gene co-expression module detection using WGCNA}

With 200 DE genes from QN + SVA data, unsigned coexpression network was constructed using the WGCNA package in R [37]. Since we used differentially expressed genes, prior to constructing networks, the effect of phenotype (epithelial and mesenchymal) from each gene was removed using a linear model.

$$
E_{i}=\mu+\beta_{1} \cdot P_{i}+\epsilon
$$

where, $\mu$ is the mean effect, $E_{i}$ is the expression of a gene in sample $i, \beta_{1}$ is the regression coefficient of phenotype, $P_{i}$ is the phenotype label for sample $i$ and $\epsilon \sim N(0,1)$. Expression of gene $\widehat{E}$ after regressing out effect of phenotype is given by:

$$
\widehat{E}_{i}=E_{i}-\left(\mu+\beta_{1} \cdot P_{i}\right)
$$

Next using this, we computed an adjacency matrix $a_{i j}$ using pearson correlation:

$$
a_{i j}=\left|\operatorname{corr}\left(e_{i}, e_{j}\right)\right|^{\beta}
$$

where $e_{n}$ is the expression of gene $n$ and $\beta$ is the softthresholding power for weighted networks. Best scalefree topology fitting index $R^{2}$ was obtained at $\beta=5.5\left(R^{2}=\right.$ 0.77). The adjacency matrix was then transformed to a topological overlap based similarity matrix given by:

$$
T O M_{i j}=\sum_{u} \frac{\sum_{k} a_{i k} a_{k j}+a_{i j}}{\min \left\{\sum_{k} a_{i k}, \sum_{k} a_{j k}\right\}+1-a_{i j}}
$$

The topological overlap between two nodes is the measure of relative interconnectedness. The TOM was then transformed to dissimilarity matrix:

$$
\operatorname{diss} \mathrm{TOM}_{i j}=1-\mathrm{TOM}_{i j}
$$

Genes were then clustered using average linkage hierarchical clustering.

Co-expression modules were derived from clustering dendrogram using Dynamic Tree Cut with hybrid method. This helped overcome the need for manually selecting a cut-off height. We set minimum module size to 15 since we were looking for modules among 200 genes. The expression profile of each module is represented by its eigengene, which is the first principal component of the module.

\section{RT-qPCR}

RNA was isolated from cells at $\sim 80 \%$ confluency using RNeasy kit (Qiagen) and subsequent cDNA libraries were prepared using Bio-Rad cDNA synthesis kit. TaqMan gene expression assays were used to determine mRNA expression levels using the following probes: $\beta$-actin Hs_1060665_g1, LSR Hs01076319_g1, S100A14 Hs04189107, DPYSL3 Hs00181665_m1, C1orf116 Hs00 539900_g1, OVOL1 Hs00970334, CDH1 Hs01023894, CDH2 Hs00983056_m1, ZEB1 Hs00232783_m1.

Relative Expression Calculations: In the qPCR, the target of interest in each sample is measured using at least three biological replicates. The $\mathrm{Ct}$ value for each biological replicate is calculated as an average of three technical replicates. Then the $\mathrm{Ct}$ value of each biological replicate is normalized to $\beta$-actin by subtracting it from the corresponding $\mathrm{Ct}$ value of $\beta$-actin $(-\Delta \mathrm{Ct})$. The two groups of interest are compared using a Student's t-test. The values plotted in the graph are the average of the base 2 anti-log transformations of $-\Delta C t$ for the biological replicates of interest divided by the average of the base 2 anti-log of $-\Delta \mathrm{Ct}$ for the reference group. The standard errors of the mean are determined from biological replicates.

\section{Western blot}

Protein extracts were prepared using Frackleton-lysis buffer with protease inhibitors (Thermo Scientific $78,410)$, and samples were electrophoresed on $4-15 \%$ SDS-PAGE (Bio-Rad), transferred to a nitrocellulose membrane and blocked with casein blocking buffer (Sigma B6429). The list of antibodies used for western blotting is in Additional file 2: Table S6. The Licor 
Odyssey fluorescence scanner was used for visualizing the westerns.

\section{siRNA knockdown of C1orf116}

C1orf116 siRNA (ThermoFisher, cat\#: 4,392,420) with RNAiMAX transfection reagent (ThermoFisher) was used for siRNA transfections. Some alterations were made to manufacturer's recommended protocol. Cells were seeded at a density result in 50\% confluency the following day. Using a 6 well plate, 9 ul of RNAiMAX reagent and $3 \mathrm{ul}(30 \mathrm{pmol})$ of siRNA (each diluted in 150 ul of Opti-MEM media) was added to each well the day after seeding. $72 \mathrm{~h}$ later RNA was isolated (Qiagen, Rneasy mini kit) from plates and gene expression was analyzed.

\section{C1orf116 expression in cancer patient data}

We identified publicly available published cancer patient (breast, prostate, esophageal, liver, colorectal, and lung) gene expression studies with at least 150 patients on Oncomine [38]. Gene expression data for studies (GSE17536 [39], GSE11121 [40], GSE25066 [41], GSE22358 [42], GSE7390 [43], GSE68465 [44], GSE31210 [45], and GSE21034 [46]) available on GEO were obtained using the GEOquery R package [47]. Probeset IDs corresponding to C1orf116 were used. Gene level expression was obtained by aggregating multiple probe expression values with median. Wilcoxon rank sum test was used to test association between expression of C1orf116 and grade, smoking status and cancer sample site. We also looked at association between tumor grade and C1orf116 expression in 4 breast cancer, 1 colorectal cancer and 1 lung cancer studies from Oncomine. We adjust Wilcoxon rank sum $p$-values with bonferroni correction for a total of 23 tests performed for clinical associations (Table 2, Additional file 3: Table S7 and Additional file 4: Figure S7).

\section{Results}

We identified publically available gene expression microarray datasets that queried gene expression of cell lines induced to undergo EMT [7-21]. We confirmed the phenotype of the samples by referring to associated publications for immunohistochemistry staining and/or protein expression of known epithelial or mesenchymal markers (Table 1, Additional file 2: Table S1). 95 cell line observations (45 unique samples and 50 replicates) from 15 datasets that showed sufficient evidence of correct phenotypic labeling included 49 cell lines of epithelial phenotype and 46 cell lines of mesenchymal phenotype.

\section{Normalization methods show consistency in signal}

Technical variability in the form of noise and batch-effects is inherent in gene expression data. We performed
Table 2 Association of C1orf1 16 expression in lung and prostate cancer patients

\begin{tabular}{lcc}
\hline Test group & $\begin{array}{c}\text { Wilcoxon rank } \\
\text { sum } p \text {-value }\end{array}$ & $\begin{array}{c}\text { Bonferroni } \\
\text { adjusted } \\
p \text {-value }\end{array}$ \\
\hline Lung cancer (Director's Lung Challenge): grade [44] & \\
Grade1 vs Grade 2 & $1.4191 \mathrm{e}-06$ & $3.27 \mathrm{E}-05$ \\
Grade 2 vs Grade 3 & $1.1481 \mathrm{e}-10$ & $2.65 \mathrm{E}-09$ \\
Grade 1 vs Grade 3 & $2.6121 \mathrm{e}-17$ & $6.00 \mathrm{E}-16$ \\
Lung cancer (Director's Lung Challenge): Smoking Status [44]
\end{tabular}

rigorous confounding factor correction to make gene expression comparisons between epithelial and mesenchymal samples that came from different studies, platforms, and cell lines. We used simple normalization methods including column standardized mean centered (MCtr) [35] and Quantile Normalization (QN) [33] and more rigorous methods that included Surrogate Variable Analysis (SVA) [34] and combination of QN followed by SVA (QN + SVA). With each normalization method (MCtr, QN, SVA, QN + SVA), we compared mean expression of epithelial and mesenchymal cell lines by a two-sample t-test for differential expression. We evaluated concordance among normalization methods to determine signal robustness - any individual method may be subject to false positives due to different patterns such as outliers, batch effects, etc. For this, we restricted our analysis to 7276 genes that were common across all studies. We used spearman correlation to test association between raw test statistics ( $n=7276$ genes) obtained from two-sample t-test from each of type of normalized data. Test-statistic distributions from individual normalization methods were significantly correlated with each other ( $p$-value <2.2e-16, $n=7276$ ). This indicates that signal produced by data normalized using a particular method is consistent with others (Fig. 3, Additional file 5: Figure S1, Additional file 6: Figure S2 and Additional file 7: Figure S3).

Next, to assess if normalization improved overall grouping of epithelial and mesenchymal phenotypes together, we clustered samples from each of the normalized datasets using hierarchical clustering (using all 7276 genes). Next, to evaluate this grouping we used the Baker Hubert Index $(\mathrm{BH})$ with known phenotype vector as group assignments. Values of the $\mathrm{BH}$ index range 


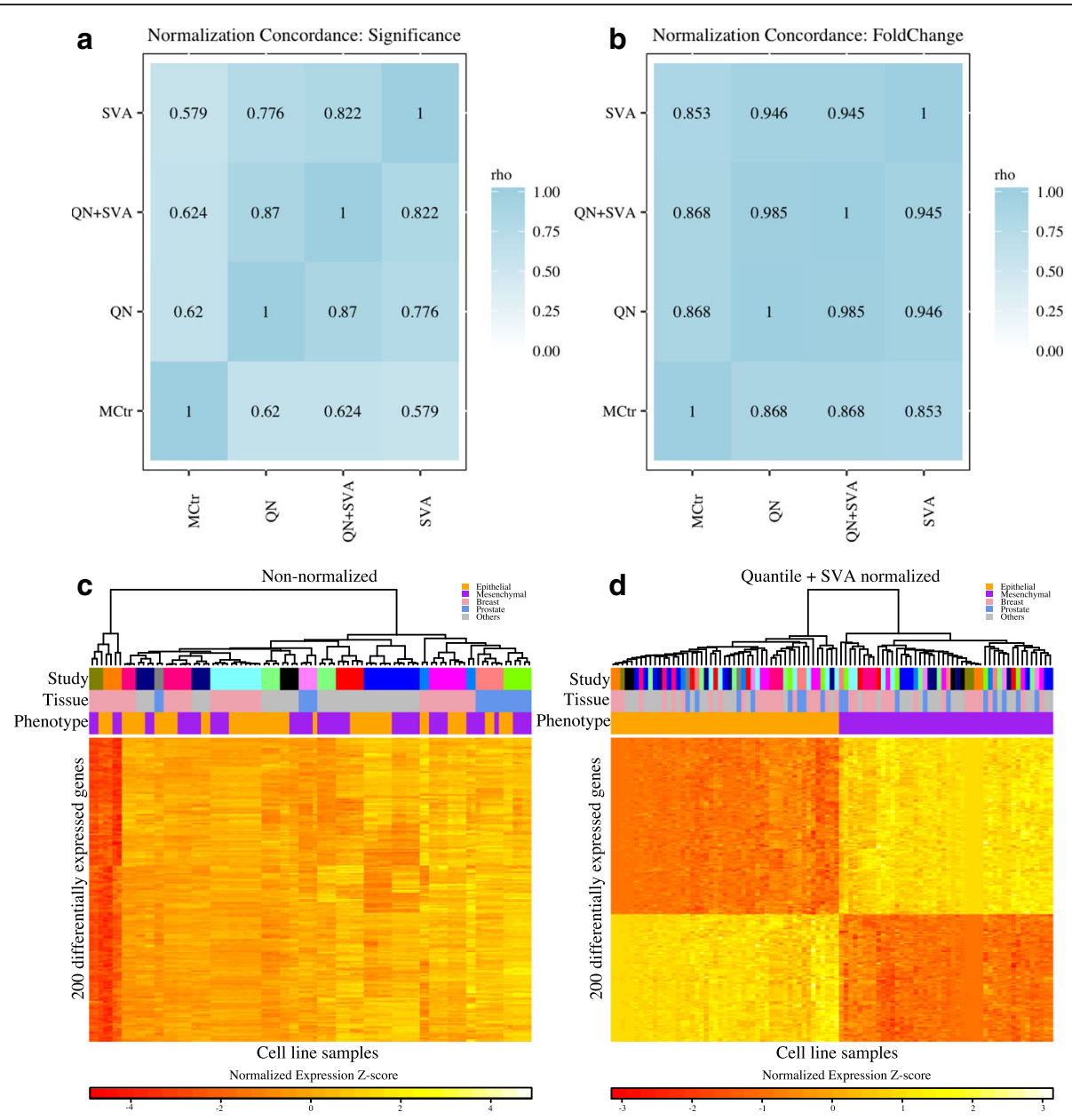

Fig. 3 Consistency in differential expression signal across normalization methods. a Correlation heatmap showing concordance (Spearman rho) among ranks of differentially expressed genes using the four normalization methods ( $n=7276)$. Genes were ranked by raw t-test $p$-values. b Correlation heatmap showing concordance (Spearman rho) among fold-change of differentially expressed genes using the four normalization methods ( $n$ = 7276). c Hierarchical Clustering of top 200 differentially expressed genes with uncorrected data shows strong clustering of samples by study rather than by phenotype. $\mathbf{d}$ Hierarchical Clustering of top 200 differentially expressed genes with QN + SVA (Quantile Normalized + SVA) corrected data clusters by epithelial and mesenchymal phenotype

from -1 to 1 , with larger values indicating better grouping [48]. Table 3 shows that grouping of samples by phenotype (epithelial or mesenchymal) is considerably improved in normalized datasets in comparison to nonnormalized data. QN + SVA performs the best, followed by SVA, MCtr and QN.

\section{Differential expression analyses reveal universal EMT genes across multiple carcinoma types}

With every form of normalized data (MCtr, QN, SVA, $\mathrm{QN}+\mathrm{SVA}$ ), we determined differentially expressed genes between epithelial and mesenchymal cell phenotype by a two-sample t-test. A gene list ranked by raw $p$-values from the $\mathrm{t}$-test was generated for each normalization method. Assuming equal likelihood of error in correction methods (Fig. 2), for each gene we assigned a differential rank that was the average of $p$-value ranks from all four normalization methods. This was used to generate a final integrated ranked gene list (Additional file 8: Table S2).

We defined a candidate universal EMT gene list by the top 200 genes from the integrated gene list (absolute fold change $>1.2$ and FDR $<0.005$ in SVA, QN + SVA and MCtr normalized data) (Additional file 8: Table S2). These genes are representative of global differential

Table 3 Evaluation of sample grouping (with 7276 genes) using Baker Hubert index and phenotype information

\begin{tabular}{|c|c|c|c|c|c|}
\hline & No normalization & $\begin{array}{l}\text { Quantile Normalization } \\
\text { (QN) }\end{array}$ & $\begin{array}{l}\text { Surrogate Variable } \\
\text { Analysis (SVA) }\end{array}$ & $\mathrm{QN}+\mathrm{SVA}$ & $\begin{array}{l}\text { Median Centered } \\
\text { Column Scaled }\end{array}$ \\
\hline Baker Hubert Index & 0.0001 & 0.047 & 0.864 & 0.7995 & 0.0705 \\
\hline
\end{tabular}


EMT patterns independent of cell line origin and treatment modality.

Cancer cells recruit developmental pathways and processes to acquire migratory and invasive properties. To determine if the candidate gene list contained groups of genes working together and shared common biological functions we tested enrichment it's enrichment for Hallmark genesets (MSigDB) defined and curated by the Broad Institute [49] using a right-tailed Fisher's exact test. The most significantly enriched gene set was epithelial to mesenchymal transition (Odds ratio $=18.3575636$, $\mathrm{FDR}=4.92 \mathrm{E}-31)$. Among the other hallmark gene sets, we found increased representation (FDR $<10 \%$ ) of several EMT related pathways including estrogen responsive genes (early and late), genes upregulated in response to low oxygen levels (hypoxia) and others [5, 50-57] (Table 4). We also found that specific estrogen responsive genes (early and late) were differentially expressed even when restricted just to the prostate cancer samples (Additional file 9: Figure S6) indicating this enrichment was not due exclusively to breast cancer cell lines in our combined analysis. When tested for GO biological processes, we found enrichment (FDR < 10\%) for several developmental terms including epidermis development, anatomical structure morphogenesis and organ development
(Additional file 10: Table S3). This further confirms that our analyses capture comprehensive signals in identifying changes in gene expression patterns across cancer types during EMT.

Among genes on our candidate gene list, we found known epithelial- and mesenchymal-specific genes such as E-cadherin (CDH1), Zinc Finger E-Box Binding Homeobox 1 (ZEB1), Vimentin (VIM), Transforming Growth Factor, Beta 1 (TGFB1), Tissue Inhibitor Of Metalloproteinase 1 (TIMP1) [5, 58], N-cadherin (CDH2) (Table 5). We also observed enrichment of collagen genes that are known to be associated with cell adhesion and migration amongst DE genes (Fisher's exact $p$-value 1.124e-05) [5]. In addition, we also found known EMT related transcription factors such as ZEB1, ETS1 and LSR in our candidate gene list.

We also compared our list of genes to the core EMT gene signature described by Groger et al. [27]. We found 43 common genes from their study (Additional file 11: Table S4). These included genes such as $C D H 1, C D H 2$, $V I M, L S R$ and some collagen genes. Several known EMT genes such as TGFB, TIMP1, ETS1 that were found in universal EMT genes were missing from their list. Some other genes such as S100A14, DPYSL3 and C1orf116 (Additional file 12: Figure S4 and Additional file 13: Figure S5) that we validate as differential EMT genes in our study, were also not found in their core gene list.

Table 4 Enriched MsigDB Hallmark genesets

\begin{tabular}{|c|c|c|c|c|}
\hline Geneset & $p$-values & oddsratio & FDR & Genes in set \\
\hline $\begin{array}{l}\text { HALLMARK Epithelial mesenchymal } \\
\text { transition }\end{array}$ & $9.84 \mathrm{E}-33$ & 18.3575636 & $4.92 \mathrm{E}-31$ & $\begin{array}{l}\text { CD59, CDH11, CDH2, COL1A1, COL1A2, COL4A2, COL5A1, } \\
\text { COL6A3, CTGF, CYR61, DAB2, DPYSL3, EDIL3, EMP3, ENO2, } \\
\text { FAP, FBN1, FBN2, FERMT2, GEM, GJA1, GREM1, LGALS1, } \\
\text { LOX, MMP14, MMP2, PCOLCE, PCOLCE2, PLAUR, PLOD1, } \\
\text { PMP22, POSTN, SERPINE1, SERPINE2, SLIT2, SPARC, SPOCK1, } \\
\text { TGFB1, TIMP1, VCAN, VIM, WNT5A }\end{array}$ \\
\hline HALLMARK Estrogen response late & 9.36E-06 & 4.332224532 & 0.00019652 & $\begin{array}{l}\text { ALDH3A2, ASS1, CDH1, CELSR2, LLGL2, LSR, MAPK13, PLXNB1, } \\
\text { RAPGEFL1, SCNN1A, SLC22A5, SLC27A2, ST14, TOB1, TRIM29 }\end{array}$ \\
\hline HALLMARK Apical junction & $1.18 \mathrm{E}-05$ & 4.516129032 & 0.00019652 & $\begin{array}{l}\text { AKT3, CDH1, CDH11, CLDN7, FBN1, GRB7, JAM3, JUP, MAPK13, } \\
\text { MMP2, MPZL2, PVRL3, SLIT2, VCAN }\end{array}$ \\
\hline HALLMARK UV response dn & $8.16 \mathrm{E}-05$ & 4.23768997 & 0.001019448 & $\begin{array}{l}\text { AKT3, COL1A1, COL1A2, CYR61, DAB2, FZD2, GJA1, HAS2, } \\
\text { KCNMA1, MAP1B, PMP22, SERPINE1 }\end{array}$ \\
\hline HALLMARK Estrogen response early & 0.000247578 & 3.495078664 & 0.002475779 & $\begin{array}{l}\text { AQP3, CELSR2, CLDN7, ELF3, GJA1, KRT15, PMAIP1, RAPGEFL1, } \\
\text { SCNN1A, SLC22A5, SLC27A2, TOB1, WWC1 }\end{array}$ \\
\hline HALLMARK Hypoxia & 0.000436298 & 3.276838008 & 0.003635818 & $\begin{array}{l}\text { AKAP12, CHST2, COL5A1, CTGF, CYR61, ENO2, ETS1, HMOX1, } \\
\text { KDELR3, LOX, PLAUR, SERPINE1, SRPX }\end{array}$ \\
\hline HALLMARK Inflammatory response & 0.000679488 & 3.786760716 & 0.004246802 & $\begin{array}{l}\text { CD70, CHST2, EMP3, FZD5, HAS2, HRH1, MMP14, PLAUR, } \\
\text { SERPINE1, TIMP1 }\end{array}$ \\
\hline HALLMARK KRAS signaling up & 0.00061698 & 3.554348835 & 0.004246802 & $\begin{array}{l}\text { AKAP12, EPB41L3, ETS1, GFPT2, GNG11, JUP, MAP7, MPZL2, } \\
\text { PLAUR, TMEM158, TRIB2 }\end{array}$ \\
\hline HALLMARK Angiogenesis & 0.003822541 & 7.2 & 0.02123634 & JAG2, POSTN, TIMP1, VCAN \\
\hline HALLMARK Complement & 0.00451196 & 3.068992514 & 0.022559801 & $\begin{array}{l}\text { CD59, COL4A2, CTSD, MMP14, PLAUR, SERPINE1, TIMP1, } \\
\text { TIMP2, ZEB1 }\end{array}$ \\
\hline HALLMARK Myogenesis & 0.00594623 & 2.929880329 & 0.027028319 & $\begin{array}{l}\text { COL1A1, COL4A2, COL6A3, ERBB3, MEF2C, NCAM1, PDLIM7, } \\
\text { SPARC, TGFB1 }\end{array}$ \\
\hline HALLMARK TGF beta signaling & 0.010673511 & 4.097902098 & 0.044472964 & BCAR3, CDH1, SERPINE1, SMURF2, TGFB1 \\
\hline
\end{tabular}


Table 5 Rank of known epithelial and mesenchymal specific genes and DE genes found in Hallmark Epithelial to mesenchymal transition [73]

\begin{tabular}{|c|c|c|c|c|c|}
\hline Gene Symbol & $\begin{array}{l}\text { Order in } \\
\text { average } \\
\text { rank }\end{array}$ & $\begin{array}{l}\text { Gene } \\
\text { Symbol }\end{array}$ & $\begin{array}{l}\text { Order in } \\
\text { average } \\
\text { rank }\end{array}$ & $\begin{array}{l}\text { Gene } \\
\text { Symbol }\end{array}$ & $\begin{array}{l}\text { Order in } \\
\text { average } \\
\text { rank }\end{array}$ \\
\hline EMP3 & 3 & PCOLCE2 & 45 & $V I M^{*}$ & 144 \\
\hline VCAN & 4 & FAP & 54 & FERMT2 & 147 \\
\hline GEM & 6 & $\mathrm{CDH} 11$ & 73 & POSTN & 150 \\
\hline $\mathrm{CDH} 2^{*}$ & 11 & TGFB1* & 81 & FBN2 & 155 \\
\hline$Z E B 1^{*}$ & 12 & SPARC & 84 & GJA1 & 159 \\
\hline SPOCK1 & 13 & CYR61 & 90 & SERPINE1 & 161 \\
\hline COLAA2 & 14 & WNT5A & 95 & $D A B 2$ & 168 \\
\hline FBN1 & 15 & CD59 & 98 & COLIA1 & 171 \\
\hline PMP22 & 21 & GREM1 & 106 & MMP2 & 174 \\
\hline COL5A1 & 22 & PLAUR & 108 & PCOLCE & 181 \\
\hline $\mathrm{CDH} 1^{*}$ & 24 & CTGF & 118 & ENO2 & 187 \\
\hline SLIT2 & 33 & COLIA2 & 120 & LGALSI & 191 \\
\hline EDIL3 & 36 & PLOD1 & 124 & SERPINE2 & 162 \\
\hline DPYSL3 & 42 & MMP14 & 127 & & \\
\hline COL6A3 & 43 & LOX & 129 & & \\
\hline
\end{tabular}

*commonly used EMT marker genes

\section{Candidate gene list identified genes previously unknown in prostate cancer EMT}

In addition to genes well established in the process of EMT, we also identified genes that had only been described in EMT in a subset of cancer types, including two epithelial specific genes, lipolysis stimulated lipoprotein receptor $(L S R)$ and $S 100$ calcium binding protein A14 (S100A14), and one mesenchymal specific gene, dihydropyrimidinase-like 3 (DPYSL3). Previous studies have investigated role of $L S R$ in breast cancer EMT [59], and S100A14 has been examined in pancreatic and cervical cancer $[60,61]$. Previous studies have indicated involvement of DPYSL3 in malignant pancreatic and gastric tumors [62, 63].

We validated the expression of these genes in an in vitro model of prostate cancer EMT. mRNA and protein expression levels of these genes were determined in one epithelial and two mesenchymal prostate cancer cell line PC3 derivatives. PC3-Epi is an expansion of a highly epithelial clone from the parental PC3 population. The mesenchymal derivatives were generated from PC3 cells by M2 macrophage co-cultures (PC3-EMT) and Taxol treatment and subsequent resistance (PC3-TxR) [20, 64]. RT-qPCR of canonical epithelial and mesenchymal genes, OVOL1, OVOL2, CDH1, ZEB1, and CDH2, confirmed the appropriate phenotypic states for these cells lines (Fig. 4a). Elevated levels of S100A14 mRNA was observed in PC3-Epi compared to mesenchymal PC3-
EMT and PC3-TxR. Similarly, mRNA expression of epithelial gene $L S R$ was found to be higher in PC3-Epi than in its mesenchymal counterparts, PC3-EMT and PC3TxR (Fig. 4b).

Conversely, the mesenchymal gene DPYSL3 was extremely upregulated in PC3-EMT and PC3-TxR than in PC3-Epi (Fig. 4b). These results were supported by western blot analysis, which demonstrated protein levels mirrored the mRNA expression (Fig. 4c).

\section{C1orf116 was discovered to be a novel EMT regulator}

Our candidate gene list also contained genes that have not been previously described as related to the EMT process in any cancer type or in any physiologic process. One of these novel candidate EMT genes, C1orf116 (also known as $S A R G$ ), is a poorly characterized gene with only one PubMed listed publication [65]. We first validated our finding from microarray data using the PC3 in vitro model of EMT and found increased mRNA expression in PC3-Epi cells compared to PC3-emt (1.3 fold) and PC3-TxR (8.8 fold). These results were supported by elevated protein expression of C1orf116 in PC3-epi cells (Fig. 5a-b).

Increased expression C1orf116 in epithelial cells confirmed of it as an epithelial marker gene. We applied gene network analysis [37], that revealed weighted coexpression gene modules (groups of co-expressed genes) and showed that C1orf116 clustered with other epithelial genes including CDH1, LSR, S100A14 and others (Additional file 14: Table S5, Fig. 6). LSR and S100A14 were among the known-unknown genes whose expression was validated in PC3 cell lines. This confirmed its association with other epithelial genes universal across other disease types. Through manual literature search, we identified that a subset of the C1orf116 module gene list have been shown to be associated with multiple cancer types. Among other genes in the modules, $S H 2 D 3 A$, $A P 1 M 2, C D S 1$ and SCNN1A haven't been previously studied in cancer biology. This shows that in addition to being a novel EMT regulator in prostate cancer, C1orf116 could have broad effects across multiple cancer types.

Next, we interrogated the possible role of C1orf116 in in vivo malignant progression. For this, we identified gene expression studies with at least 150 patients that also had information on tumor grade and expression data for C1orf116 and were able to find breast, prostate, colorectal and lung cohorts (Additional file 4: Figure S7). We found that C1orf116 expression is decreased in metastatic lesions compared to localized tumors in prostate cancer patients (Fig. 7a) [46]. Likewise, C1orf116 expression decreased with increasing cancer grade in patients with lung cancer (Fig. 7b) [44]. Studies have shown that lung cancer patients with history of smoking 


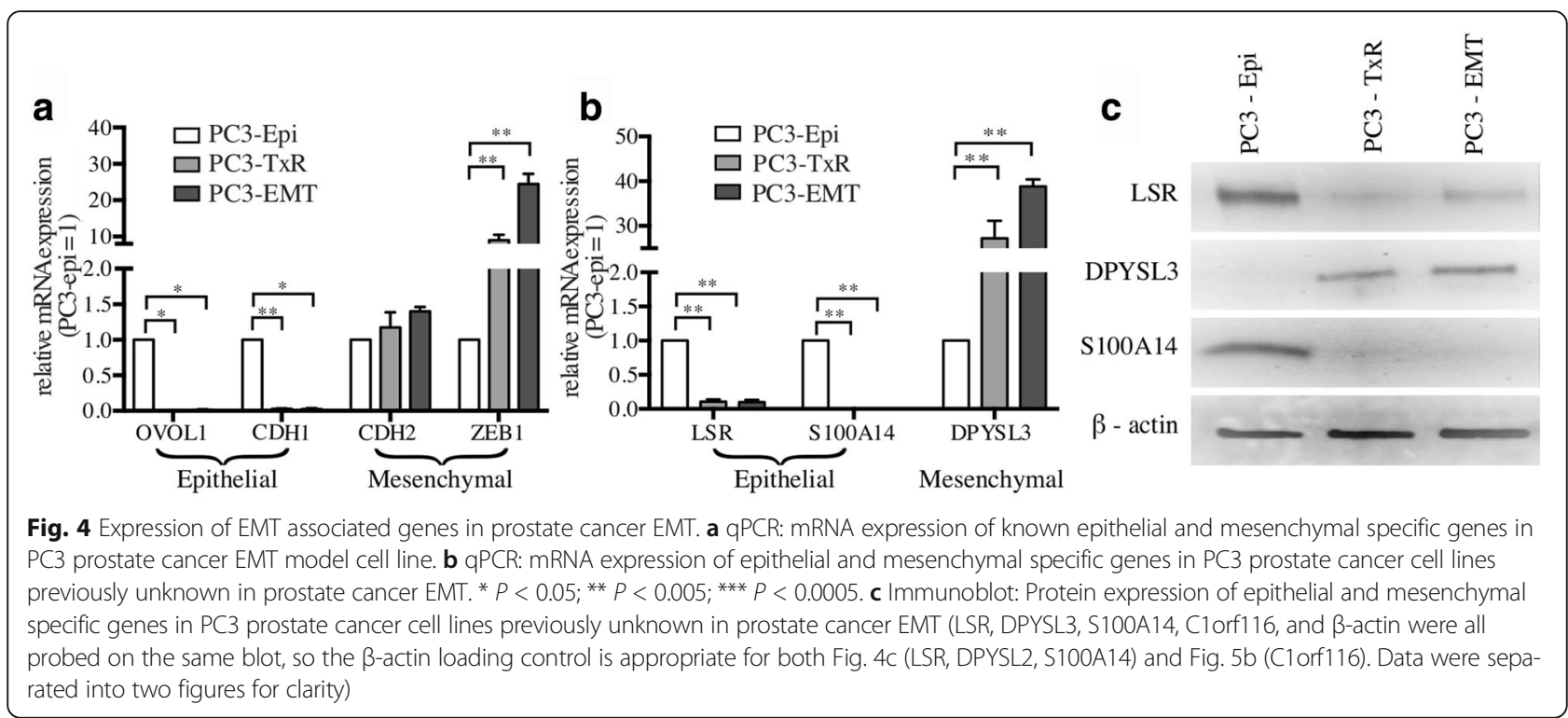

tobacco/cigarette exhibit lower expression levels of Ecadherin and higher levels of mesenchymal markers such as vimentin [66, 67]. Previous studies have also indicated that cigarette smoking can induce EMT in non-small cell lung cancer [68]. Analogous to these findings, we observed reduced expression of C1orf116 among lung cancer patients with smoking habits (Fig. 7c-d) [44, 45]. In some breast cancer datasets expression of C1orf116 increased with increasing cancer grade (Additional file 3: Table S7 and Additional file 4: Figure S7). This suggested that in addition to expression changes in in vitro cell line models, changes in C1orf116 expression could potentially have a functional role in clinically-important disease progression in cancer patients.

To test the role of C1orf116 as a driver of an epithelial phenotype, we used siRNA-mediated knockdown of the gene in PC3-Epi cells. We found that siRNA-mediated knockdown of C1orf116 expression resulted in decreased expression of epithelial markers OVOL1, ESRP1, and $C D H 1$, and increased expression of mesenchymal marker $\mathrm{CDH} 2$ (Fig. 5c). This suggests that C1orf116 plays a functional role in maintaining epithelial phenotype. Significant upregulation of mesenchymal genes in response to C1orf116 knockdown indicates it as a novel regulator of EMT.

\section{Discussion}

EMT may be an early step in cancer metastasis and has been associated with chemoresistance and disease progression $[69,70]$. Though EMT is common among all solid tumor types and is essential in early development, common drivers of EMT across multiple cancer types have not been described. Several studies have investigated EMT in cell lines from within a single disease type. Although most studies have been confined to very
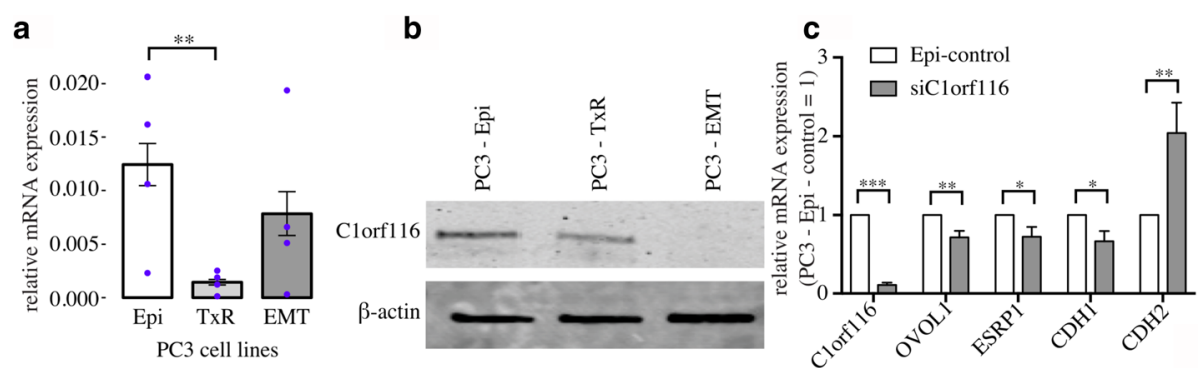

Fig. 5 C1orf1 16: a novel EMT regulator. a qPCR: mRNA expression of C1orf1 16 in EMT model prostate cancer cell lines PC3-Epi, PC3-EMT and PC3-TxR ${ }^{*} P<0.1$; ${ }^{*} P<0.05$; ${ }^{* *} P<0.005$. b Immunoblot: Protein expression of C1 orf1 16 in EMT model prostate cancer cell lines PC3-Epi, PC3-EMT and PC3TxR (LSR, DPYSL3, S100A14, C1orf116, and $\beta$-actin were all probed on the same blot, so the $\beta$-actin loading control is appropriate for both Fig. 4C (LSR, DPYSL2, S100A14) and Fig. 5b (C1orf116). Data were separated into two figures for clarity). c qPCR: mRNA expression of C1orf116 and other known epithelial (OVOL1, ESRP1 and CDH1) and mesenchymal (CDH2) gene in PC3-Epi cells transfected with C1orf116-siRNA relative to empty vector control ${ }^{*} P<0.1$; ${ }^{*} P<0.05$; ${ }^{* * *} P<0.005$ 


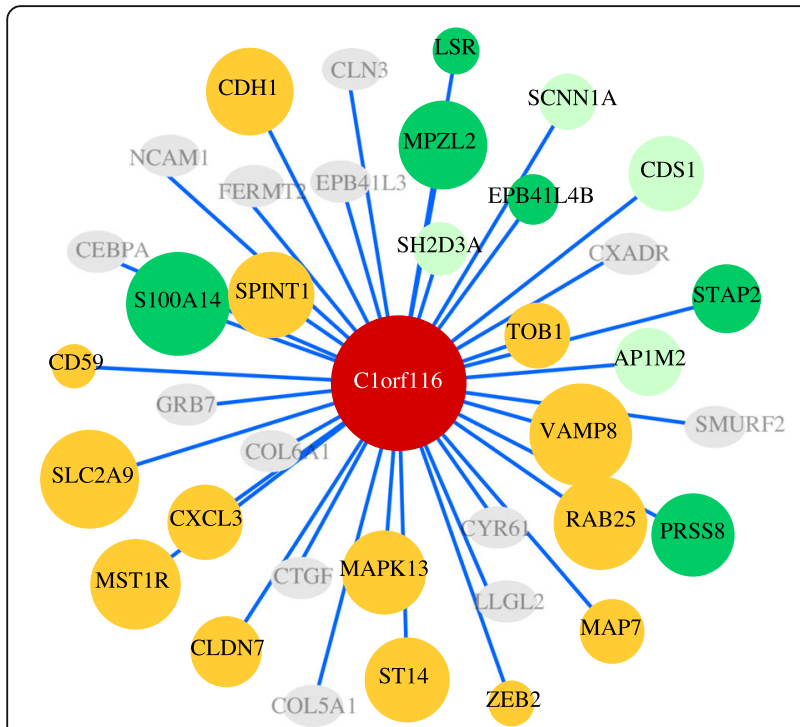

Fig. 6 Clorf1 16 associated genes in weighted gene correlation network module. This correlation network shows association of C1orf116 module genes obtained from WGCNA. Node size is a function of correlation with C1orf116 expression. Yellow nodes represent genes that have been previously studied in multiple (greater than 3 ) cancer types. Bright green nodes are the genes that have been studied in 3 or less cancer types. Light green nodes are genes that have not been specifically studied in cancer. Gray nodes were genes that were not significantly associated with expression of Clorf116

small sample size. To address this, we systematically integrate multiple EMT studies to increase power and identify novel drivers of EMT universal to all cancer types.

A significant challenge in multi-study analysis comes from various sources of heterogeneity arising from study specific technical and biological variation. Biological variation interferes with analyses, especially when it is not the signal of interest. We employed two strategies to address various sources of heterogeneity and noise. First, we chose stringent normalization methods that have been shown to reduce the influence of such heterogeneity (SVA, quantile normalization, and scaled median centering). We recognize that these methods may have their failure modes and limitations. Therefore, we defined our final differentially expressed gene list from consensus ranking across all four normalization schemes. Thus even if a single method introduced an error or failed to account for a particular effect, the final gene list may be more robust than results from any individual method. However, technical variation and experimental heterogeneity may still influence the results of our analysis, as no method has been shown to fully remove such effects from expression data. Therefore, experimental validation and comparison with external functional annotation were important.

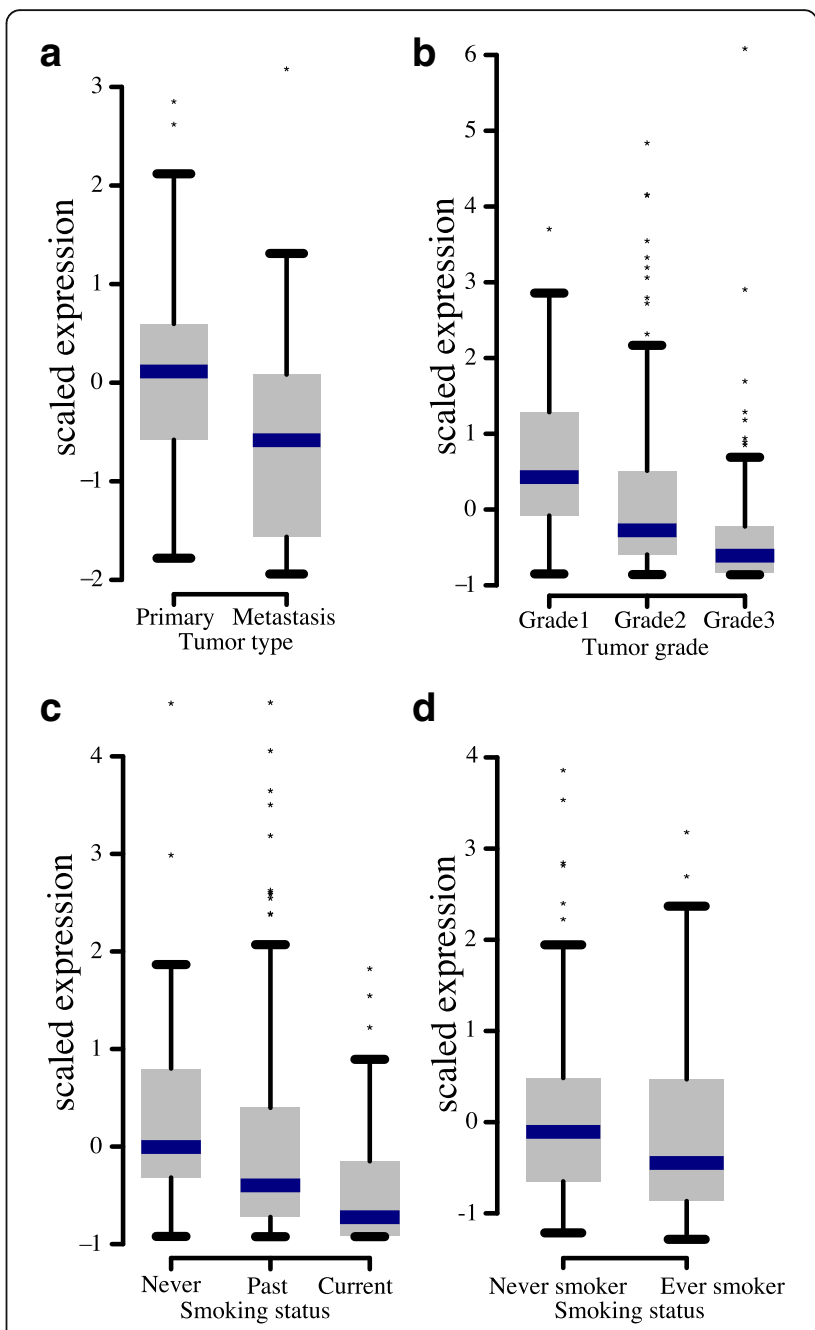

Fig. 7 C1orf116 expression in cancer patient data. a Decreased expression of C1orf116 is seen in metastatic tumor type compared to primary prostate cancer (Taylor dataset); unadjusted $P=0.0340$, Bonferroni adjusted $P=0.51$. $\mathbf{b}$ Expression of C1 orf116 decreases in high grade lung cancer (Director's challenge dataset); Bonferroni adjusted $P<0.0005$. c Clorf116 is downregulated in lung cancer patients with increased smoking habits (Director's challenge dataset); unadjusted $P<0.01$, Bonferroni adjusted $P<0.1$. d C10rf116 is downregulated in lung cancer patients with smoking habits in comparison to non-smokers (Okayama dataset); unadjusted $P=0.0586$, Bonferroni corrected $P=0.879$

Integrating across multiple studies did improve power and helped us detect novel genes that showed consistent effect across multiple studies, which could be concealed in a single study. We found three groups of genes in the EMT differentially expressed list: a) known EMT genes (e.g. CDH1, ZEB1, TGFB, CDH2, VIM, TIMP1), b) EMT genes previously unknown in prostate cancer (LSR, S100A14, DPYSL3) and c) novel EMT genes (including C1orf116).

We confirmed our discovery of unknown EMT genes in prostate cancer by testing expression of $L S R$, S100A14, and DPYSL3 in a PC3 prostate cancer cell line 
model of EMT. Previous studies have shown that LSR suppresses EMT phenotype in claudin-low breast cancer cell lines [59]. S100A14 has been studied in breast cancer progression and is showed to be involved in EMT in human cervical and pancreatic cancer cells $[60,61,71]$. DPYSL3 is associated with malignant gastric and pancreatic tumors $[62,63]$. Moreover studies suggest that mRNA expression of DPYSL3 is positively correlated with Vascular Endothelial Growth Factor (VEGF), a gene thought to be involved in EMT [72]. This data indicates that our method bridged EMT cancer biology across different disease types and captures global expression patterns in EMT (Additionale file 12: Figure S4A-C).

We confirmed discovery of C1orf116 as epithelial specific gene by testing its expression in PC3 in vitro model of EMT. siRNA knockdown of C1orf116 in PC3 epithelial cell lines showed loss of epithelial markers and gain of mesenchymal markers thereby confirming its functional role as a negative driver of EMT. Clinical data from breast, prostate cancer and lung cancer patients also suggested that changes in expression of Clorf116 could have functional implications in disease progression.

Altogether, through this study we have found genes whose effects are represented by multiple cancer types (breast, prostate, liver, colon, esophagus and retinal pigment). We have also validated expression of some genes in an in vitro prostate cancer cell line model and potential relevance in vivo data from three tissues, including one (lung) that was not represented among our cell line data. However, these effects might not necessarily be extrapolated for cancer types not included in this study. As data become available for other tissues and cancers, further analysis can be performed.

\section{Conclusions}

Using multi-study integration approach, we identified consensus ranked universal EMT genes. This gene list comprised of a) known EMT genes that included $C D H 1$, $Z E B 1$ and $C D H 2$ b) genes studied in a subset of carcinomas, unknown in prostate cancer: LSR, S100A14 and DPYSL3 and c) novel unknown EMT and cancer genes such as C1orf116. siRNA experiments indicate it to be a potential novel regulator of EMT. Patient gene expression data shows that reduced expression of C1orf116 is associated with poor prognosis in lung and prostate cancer (unadjusted Wilcoxon rank sum $p$-value $<0.05)$. In conclusion, our approach of statistical analysis and functional validation identified universal EMT genes and candidate global regulatory genes, thereby both extending current knowledge of EMT and showed preliminary evidence of disease progression in cancer.

\section{Additional files}

Additional file 1: Table S1. Dataset information - Extended table. (XLSX $33 \mathrm{~kb}$ )

Additional file 2: Table S6. List of antibodies used in immunoblot. (XLSX 36 kb)

Additional file 3: Table S7. Association of C10rf116 expression in lung and prostate cancer patients. (XLSX $9 \mathrm{~kb}$ )

Additional file 4: Figure S7. Clorf116 expression in clinical patient data from breast and colorectal cancer. (PDF $25 \mathrm{~kb}$ )

Additional file 5: Figure S1. Hierarchical Clustering of top 200 differentially expressed genes with data corrected by quantile normalization. (PDF 429 kb)

Additional file 6: Figure S2. Hierarchical Clustering of top 200 differentially expressed genes with data corrected by SVA (Surrogate Variable Analysis) normalization. (PDF $122 \mathrm{~kb}$ )

Additional file 7: Figure S3. Hierarchical Clustering of top 200 differentially expressed genes with data corrected by MCtr (Median Centered Column Scaled) normalization. (PDF $122 \mathrm{~kb}$ )

Additional file 8: Table S2. Gene ranks - fold change and significance based. (XLSX $1503 \mathrm{~kb}$ )

Additional file 9: Figure S6. Expression of Estrogen responsive genes (A) early and (B) late in prostate cancer cell line samples from integrated data. (PDF $115 \mathrm{~kb}$ )

Additional file 10: Table S3. Gene set enrichment with GO Biological Processes term. (XLSX $36 \mathrm{~kb}$ )

Additional file 11: Table S4. Common genes with Groger et al. study and 200 DE genes. (XLSX 32 kb)

Additional file 12: Figure S4. Expression of EMT genes previously unknown in prostate cancer in integrated cell lines data. Expression of LSR (A), S100A14 (B) and DPYS $\angle 3(C)$ in breast, prostate and others (retinal pigment, liver, colon and esophageal) cancer cell lines from QN + SVA normalized integrated data. (PDF $97 \mathrm{~kb}$ )

Additional file 13: Figure S5. Expression of C1orf116 in breast, prostate and others (retinal pigment, liver, colon and esophageal) cancer cell lines from integrated data. (PDF $45 \mathrm{~kb}$ )

Additional file 14: Table S5. C1orf116 module genes obtained from Weighted Gene Co-expression analysis. (XLSX 42 kb)

\section{Abbreviations}

EMT: Epithelial to mesenchymal transition; MCtr: Median Centered Column Scaled; MET: Mesenchymal to epithelial transition; QN: Quantile Normalization; RT-qPCR: Reverse transcription quantitative polymerase chain reaction; SVA: Surrogate Variable Analysis; WGCNA: Weighted Gene Coexpression Network Analysis

Acknowledgements

We would like to thank members of Battle and Pienta lab for their inputs.

\section{Funding}

K.J. Pienta is supported by the National Cancer Institute (CA093900, CA163124 U54CA143803, CA143055) and the Prostate Cancer Foundation. Alexis Battle is supported by R01 MH101814 (NIH/NIMH), R01 HG008150 (NIH/NIGMS), 1R01GM1 18568-01 (NIH/NIGMS) and R01 MH101820 (NIH). Sarah R. Amend is supported by an American Cancer Society Postdoctoral Fellowship (PF-1602501-CSM). There was no role of the funding body in the design of the study and collection, analysis, and interpretation of data and in writing the manuscript.

\section{Availability of data and materials}

All data generated or analysed during this study are included in this published article [and its supplementary information files].

\section{Authors' contributions}

PP, AB and KJP conceived and designed the study. PP performed computational analysis including normalization, differential expression, concordance and WGCNA. RT-qPCR, immunoblot and siRNA experiments were performed by $\mathrm{JH}$. PP, JH and SRA analyzed clinical patient data. PP 
wrote the manuscript. JH helped with writing the initial draft of manuscript. SRA helped with drafting of manuscript and critical reviews. AB and KJP helped with critical review of manuscript. All authors read and approved the final manuscript.

\section{Competing interests}

$A B$ holds stock in Google Inc.

\section{Consent for publication}

Not applicable.

\section{Ethics approval and consent to participate}

Not applicable.

\section{Publisher's Note}

Springer Nature remains neutral with regard to jurisdictional claims in published maps and institutional affiliations.

\section{Author details}

'Department of Computer Science, Johns Hopkins University, Baltimore, MD 21218, USA. ${ }^{2}$ Department of Urology, Johns Hopkins University School of Medicine, Baltimore, MD 21287, USA.

\section{Received: 24 August 2016 Accepted: 8 June 2017}

Published online: 26 June 2017

\section{References}

1. Mehlen P, Puisieux A. Metastasis: a question of life or death. Nat Rev Cancer. 2006;6:449-58

2. Tsai $\mathrm{JH}$, Yang J. Epithelial-mesenchymal plasticity in carcinoma metastasis. Genes Dev. 2013;27:2192-206.

3. Chaffer $C L$, Weinberg R a. A Perspective on Cancer Cell Metastasis. Science (80-. ). 2011;331:1559-64.

4. Thiery JP, Acloque H, Huang RYJ, Nieto MA. Epithelial-Mesenchymal transitions in development and disease. Cell. 2009;139:871-90.

5. Kalluri $\mathrm{R}$, Weinberg RA. The basics of epithelial-mesenchymal transition. J Clin Invest. 2009;119:1420-8.

6. Craene B De, Berx G. Regulatory networks defining EMT during cancer initiation and progression. Nat Rev Cancer Nature Publishing Group. 2013; 13:97-110.

7. Bergamaschi A, Kim YH, Kwei KA, La Choi Y, Bocanegra M, Langerød A, et al. CAMK1D amplification implicated in epithelial-mesenchymal transition in basal-like breast cancer. Mol Oncol. 2008:2:327-39.

8. Choi Y La, Bocanegra M, Kwon MJ, Shin YK, Nam SJ, Yang JH, et al. LYN is a mediator of epithelial-mesenchymal transition and a target of dasatinib in breast cancer. Cancer Res 2010;70:2296-2306.

9. Papageorgis P, Lambert AW, Ozturk S, Gao F, Pan H, Manne U, et al. Smad signaling is required to maintain epigenetic silencing during breast cancer progression. Cancer Res. 2010;70:968-78.

10. Deshiere A, Duchemin-Pelletier E, Spreux E, Ciais D, Combes F, Vandenbrouck $Y$, et al. Unbalanced expression of CK2 kinase subunits is sufficient to drive epithelial-to-mesenchymal transition by Snail1 induction. Oncogene. 2013;32: 1373-83.

11. Cai J, Guan H, Fang L, Yang Y, Zhu X, Yuan J, et al. MicroRNA-374a activates Wnt/ $\beta$-catenin signaling to promote breast cancer metastasis. J Clin Invest. 2013;123:566-79.

12. Andarawewa KL, Erickson AC, Chou WS, Costes SV, Gascard P, Mott JD, et al. Ionizing radiation predisposes nonmalignant human mammary epithelial cells to undergo transforming growth factor ??-induced epithelial to mesenchymal transition. Cancer Res. 2007;67:8662-70.

13. Joyce T, Cantarella D, Isella C, Medico E, Pintzas A. A molecular signature for epithelial to Mesenchymal transition in a human colon cancer cell system is revealed by large-scale microarray analysis. Clin Exp Metastasis. 2009;26:569-87.

14. Hwang W, Yang M, Tsai M, Lan H, Su S, Chang S, et al. SNAlL regulates interleukin-8 expression, stem celllike activity, and tumorigenicity of human colorectal carcinoma cells. Gastroenterology 2011;141:279-291, 291-5.

15. Ohashi S, Natsuizaka M, Naganuma S, Kagawa S, Kimura S, Itoh H, et al. A NOTCH3-mediated squamous cell differentiation program limits expansion of EMT-competent cells that express the ZEB transcription factors. Cancer Res. 2011;71:6836-47.
16. van Zijl F, Mall S, Machat G, Pirker C, Zeillinger R, Weinhaeusel A, et al. A human model of epithelial to mesenchymal transition to monitor drug efficacy in hepatocellular carcinoma progression. Mol Cancer Ther. 2011;10:850-60.

17. Drake JM, Strohbehn G, Bair TB, Moreland JG, Henry MD. ZEB1 enhances transendothelial migration and represses the epithelial phenotype of prostate cancer cells. Mol Biol Cell. 2009:20:2207-17.

18. Leshem O, Madar S, Kogan-Sakin I, Kamer I, Goldstein I, Brosh R, et al. TMPRSS2/ERG promotes epithelial to mesenchymal transition through the ZEB1/ZEB2 axis in a prostate cancer model. PLoS One. 2011;6:e21650.

19. Kong D, Banerjee S, Ahmad A, Li Y, Wang Z, Sethi S, et al. Epithelial to mesenchymal transition is mechanistically linked with stem cell signatures in prostate cancer cells. PLoS One. 2010;5:e12445.

20. Roca H, Hernandez J, Weidner S, McEachin RC, Fuller D, Sud S, et al. Transcription factors OVOL1 and OVOL2 induce the Mesenchymal to epithelial transition in human cancer. PLoS One. 2013;8:e76773.

21. Takahashi E, Nagano O, Ishimoto T, Yae T, Suzuki Y, Shinoda T, et al. Tumor necrosis factor-a regulates transforming growth factor- $\beta$-dependent epithelial-Mesenchymal transition by promoting Hyaluronan-CD44-Moesin interaction. J Biol Chem. 2010;285:4060-73.

22. Edgar R, Domrachev M, Lash AE. Gene expression Omnibus: NCBI gene expression and hybridization array data repository. Nucleic Acids Res. 2002;30:207-10.

23. Rung J, Brazma A. Reuse of public genome-wide gene expression data. Nat Rev Genet. 2012;14:1-11.

24. Pierre M, DeHertogh B, Gaigneaux A, DeMeulder B, Berger F, Bareke E, et al. Meta-analysis of archived DNA microarrays identifies genes regulated by hypoxia and involved in a metastatic phenotype in cancer cells. BMC Cancer. 2010;10:176.

25. Sontrop HMJ, Verhaegh WFJ, Reinders MJT, Moerland PD. An evaluation protocol for subtype-specific breast cancer event prediction. PLoS One. 2011;6:e21681.

26. Chen M, Wang K, Zhang L, Li C, Yang Y. The discovery of putative urine markers for the specific detection of prostate tumor by integrative mining of public genomic profiles. PLoS One. 2011;6:e28552.

27. Gröger CJ, Grubinger M, Waldhör T, Vierlinger K, Mikulits W. Meta-analysis of Gene expression signatures defining the epithelial to Mesenchymal transition during cancer progression. PLoS One. 2012;7:e51136.

28. Button KS. loannidis JP a, Mokrysz C, Nosek B a, Flint J, Robinson ESJ, et al. power failure: why small sample size undermines the reliability of neuroscience. Nat. Rev. Neurosci. 2013;14:365-76.

29. Zöllner S, Pritchard JK. Overcoming the Winner's curse: estimating penetrance parameters from case-control data. Am J Hum Genet. 2007;80:605-15.

30. Choi JK, Yu U, Kim S, Yoo OJ. Combining multiple microarray studies and modeling interstudy variation. Bioinformatics. 2003;19:i84-90.

31. Eklund AC, Szallasi Z. Correction of technical bias in clinical microarray data improves concordance with known biological information. Genome Biol. 2008;9:R26.

32. Leek JT. Svaseq: removing batch effects and other unwanted noise from sequencing data. Nucleic Acids Res. 2014;42:e161.

33. Bolstad BM, Irizarry RA, Åstrand M, Speed TP. A comparison of normalization methods for high density oligonucleotide array data based on variance and bias. Bioinformatics. 2003:19:185-93.

34. Leek JT, Storey JD. Capturing heterogeneity in gene expression studies by surrogate variable analysis. PLoS Genet. 2007;3:1724-35.

35. Shabalin AA, Tjelmeland $H$, Fan C, Perou CM, Nobel AB. Merging two geneexpression studies via cross-platform normalization. Bioinformatics. 2008;24: 1154-60.

36. Baker FB, Hubert LJ. Measuring the power of hierarchical cluster analysis. J Am Stat Assoc. 1975;70:31-8.

37. Langfelder P, Horvath S. WGCNA: an R package for weighted correlation network analysis. BMC Bioinformatics. 2008;9:559.

38. Rhodes DR, Yu J, Shanker K, Deshpande N, Varambally R, Ghosh D, et al. ONCOMINE: a cancer microarray database and integrated data-mining platform. Neoplasia. 2004;6:1-6

39. Smith JJ, Deane NG, Wu F, Merchant NB, Zhang B, Jiang A, et al. Experimentally derived metastasis Gene expression profile predicts recurrence and death in patients with Colon cancer. Gastroenterology. 2010;138:958-68.

40. Schmidt M, Böhm D, Von Törne C, Steiner E, Puhl A, Pilch H, et al. The humoral immune system has a key prognostic impact in node-negative breast cancer. Cancer Res. 2008;68:5405-13.

41. Hatzis C, Pusztai L, Valero V, Booser DJ, Esserman L, Lluch A., et al. A Genomic Predictor of Response and Survival Following Taxane-Anthracycline 
Chemotherapy for Invasive Breast Cancer. JAMA J. Am. Med. Assoc 2011;305: 1873-1881.

42. Glück S, Ross JS, Royce M, McKenna EF, Perou CM, Avisar E, et al. TP53 genomics predict higher clinical and pathologic tumor response in operable early-stage breast cancer treated with docetaxel-capecitabine ?? Trastuzumab Breast Cancer Res Treat. 2012;132:781-91.

43. Desmedt C, Piette F, Loi S, Wang Y, Lallemand F, Haibe-Kains B, et al. Strong time dependence of the 76-gene prognostic signature for node-negative breast cancer patients in the TRANSBIG multicenter independent validation series. Clin Cancer Res. 2007;13:3207-14.

44. Director's Challenge Consortium for the Molecular Classification of Lung Adenocarcinoma K, Shedden K, Taylor JMG, Enkemann SA, Tsao M-S, Yeatman $\mathrm{TJ}$, et al. Gene expression-based survival prediction in lung adenocarcinoma: a multi-site, blinded validation study. Nat. Med. 2008;14:822-7.

45. Okayama H, Kohno T, Ishii Y, Shimada Y, Shiraishi K, Iwakawa R, et al. Identification of genes upregulated in ALK-positive and EGFR/KRAS/ALKnegative lung adenocarcinomas. Cancer Res. 2012;72:100-11.

46. Taylor BS, Schultz N, Hieronymus H, Gopalan A, Xiao Y, Carver BS, et al. Integrative genomic profiling of human prostate cancer. Cancer Cell. 2010 18:11-22.

47. Sean D, Meltzer PS. GEOquery: a bridge between the Gene expression Omnibus (GEO) and BioConductor. Bioinformatics. 2007;23:1846-7.

48. Grimmett GR. On the number of clusters in the percolation model. J. London Math. Soc. 1976. p. 346-50.

49. Subramanian A, Tamayo P, Mootha VK, Mukherjee S, Ebert BL, Gillette MA, et al. Gene set enrichment analysis: a knowledge-based approach for interpreting genome-wide expression profiles. Proc Natl Acad Sci. 2005;102: $15545-50$.

50. Coradini D, Boracchi P, Ambrogi F, Biganzoli E, Oriana S. Cell polarity, epithelialmesenchymal transition, and cell-fate decision gene expression in ductal carcinoma in situ. Int J Surg Oncol. 2012;2012

51. Moreno-Bueno G, Portillo F. Cano a. Transcriptional regulation of cell polarity in EMT and cancer. Oncogene. 2008;27:6958-69.

52. Lamouille S, Xu J, Derynck R. Molecular mechanisms of epithelial-mesenchymal transition. Nat Rev Mol Cell Biol. 2014;15:178-96.

53. Newkirk KM, Parent AE, Fossey SL, Choi C, Chandler HL, Rajala-Schultz PJ, et al. Snai2 \{expression\} \{enhances\} \{ultraviolet\} \{radiation\}-\{induced\} \{skin\} \{carcinogenesis\}. Am J Pathol. 2007;171:1629-39.

54. Hudson LG, Choi C, Newkirk KM, Parkhani J, Cooper KL, Lu P, et al. Ultraviolet radiation stimulates expression of snail family transcription factors in keratinocytes. Mol Carcinog. 2007;46:257-68.

55. Shao DD, Xue W, Krall EB, Bhutkar A, Piccioni F, Wang $X$, et al. KRAS and YAP1 converge to regulate EMT and tumor survival. Cell. 2014;158:171-84.

56. Goswami MT, Reka AK, Kurapati H, Kaza V, Chen J, Standiford TJ, et al. Regulation of complement-dependent cytotoxicity by TGF- $\beta$-induced epithelialmesenchymal transition. Oncogene. 2016;35:1888-98.

57. Yokoyama S, Asahara H. The myogenic transcriptional network. Cell Mol Life Sci. 2011:68:1843-9.

58. Higgins DF, Kimura K, Bernhardt WM, Shrimanker N, Akai Y, Hohenstein B, et al. Hypoxia promotes fibrogenesis in vivo via HIF-1 stimulation of epithelial-tomesenchymal transition. J Clin Invest. 2007;117:3810-20.

59. Reaves DK, Fagan-Solis KD, Dunphy K, Oliver SD, Scott DW, Fleming JM. The role of lipolysis stimulated lipoprotein receptor in breast cancer and directing breast cancer cell behavior. Fanning AS, editor. PLoS One. 2014;9:e91747.

60. Wang X, Yang J, Qian J, Liu Z, Chen H, Cui Z. S100A14, a mediator of epithelial-mesenchymal transition, regulates proliferation, migration and invasion of human cervical cancer cells. Am J Cancer Res. 2015:5:1484-95.

61. Xu X, Su B, Xie C, Wei S, Zhou Y, Liu H, et al. Sonic hedgehog-Gli1 signaling pathway regulates the epithelial Mesenchymal transition (EMT) by mediating a new target gene, S100A4, in pancreatic cancer cells. PLoS One. 2014;9:996441.

62. Kawahara T, Hotta N, Ozawa Y, Kato S, Kano K, Yokoyama Y, et al. Quantitative proteomic profiling identifies DPYSL3 as pancreatic ductal adenocarcinomaassociated molecule that regulates cell adhesion and migration by stabilization of focal adhesion complex. PLoS One. 2013;8:e79654.

63. Kanda M, Nomoto S, Oya H, Shimizu D, Takami H, Hibino S, et al. Dihydropyrimidinase-like 3 facilitates malignant behavior of gastric cancer. J Exp Clin cancer Res CR. 2014;33:66.

64. Li Y, Zeng Y, Mooney SM, Yin B, Mizokami A, Namiki M, et al. Resistance to paclitaxel increases the sensitivity to other microenvironmental stresses in prostate cancer cells. J Cell Biochem. 2011;112:2125-37.
65. Steketee K. Ziel-van der Made ACJ, van der Korput HAGM, Houstmuller AB. Trapman J A bioinformatics-based functional analysis shows that the specifically androgen-regulated gene SARG contains an active direct repeat androgen response element in the first intron J Mol Endocrinol. 2004;33:477-91.

66. Vu T, Jin L, Datta P. Effect of Cigarette Smoking on Epithelial to Mesenchymal Transition (EMT) in Lung Cancer. J. Clin. Med. Multidisciplinary Digital Publishing Institute. 2016;5:44.

67. Milara J, Peiro T, Serrano A, Cortijo J. Epithelial to mesenchymal transition is increased in patients with COPD and induced by cigarette smoke. Thorax BMJ Publishing Group Ltd. 2013;68:410-20.

68. Nagathihalli NS, Massion PP, Gonzalez AL, Lu P, Datta PK. Smoking induces epithelial-to-Mesenchymal transition in non-small cell lung cancer through HDAC-mediated Downregulation of E-cadherin. Mol Cancer Ther. 2012;11: 2362-72.

69. Fischer KR, Durrans A, Lee S, Sheng J, Li F, Wong STC, et al. Epithelial-tomesenchymal transition is not required for lung metastasis but contributes to chemoresistance. Nature Nature Research. 2015:527:472-6.

70. Zheng X, Carstens JL, Kim J, Scheible M, Kaye J, Sugimoto H, et al. Epithelialto-mesenchymal transition is dispensable for metastasis but induces chemoresistance in pancreatic cancer. Nature Nature Publishing Group. 2015;527:525-30.

71. McKiernan E, McDermott EW, Evoy D, Crown J, Duffy MJ. The role of S100 genes in breast cancer progression. Tumor Biol. 2011;32:441-50.

72. Mak P, Leav I, Pursell B, Bae D, Yang X, Taglienti CA, et al. ERbeta impedes prostate cancer EMT by destabilizing HIF-1alpha and inhibiting VEGF-mediated snail nuclear localization: implications for Gleason grading. Cancer Cell NIH Public Access. 2010;17:319-32.

73. Liberzon A, Birger C. Thorvaldsd??Ttir H, Ghandi M, Mesirov JP, Tamayo P. The molecular signatures database Hallmark Gene set collection. Cell Syst. 2015:1:417-25.

\section{Submit your next manuscript to BioMed Central and we will help you at every step:}

- We accept pre-submission inquiries

- Our selector tool helps you to find the most relevant journal

- We provide round the clock customer support

- Convenient online submission

- Thorough peer review

- Inclusion in PubMed and all major indexing services

- Maximum visibility for your research

Submit your manuscript at www.biomedcentral.com/submit
Biomed Central 